\title{
Regulation of the Expression of Cytoplasmic Polyadenylation Element Binding Proteins for the Treatment of Cancer
}

\author{
YUN CHEN $^{1,2}$, YA-HUI TSAI ${ }^{1,2}$ and SHENG-HONG TSENG ${ }^{3}$ \\ ${ }^{1}$ Department of Surgery, Far Eastern Memorial Hospital, New Taipei, Taiwan, R.O.C.; \\ ${ }^{2}$ Department of Chemical Engineering and Materials Science, \\ Yuan Ze University, Taoyuan, Taiwan, R.O.C.; \\ ${ }^{3}$ Department of Surgery, National Taiwan University Hospital and National \\ Taiwan University College of Medicine, Taipei, Taiwan, R.O.C.
}

\begin{abstract}
Regulated mRNA translation plays an important role in normal cellular functions and cytoplasmic polyadenylation element binding proteins $(C P E B S)$ are the key factors that control the elongation of poly(A) tail during translation. The expression of various CPEBs has been noted to be linked to tumorigenesis, tumor growth, invasiveness and angiogenesis; however, different CPEBs appear to play diverse roles in cancer. The evidence from the literature suggests that CPEBI and $C P E B 3$ act more likely as tumor suppressors; in contrast, $C P E B 2$ and CPEB4 mainly exert oncogenic effects. In addition, different $C P E B$ subtypes may interact with each other to regulate tumorigenesis. All four CPEB mRNAs contain multiple microRNA (miRNA) binding sites, while the functions of CPEBs are regulated by various miRNAs. These results indicate that $C P E B$ s play a significant role in tumorigenesis; therefore, manipulation of the expression of different subtypes of CPEBs might modulate the behavior of cancer cells and provide new therapeutic concepts for cancer therapy. However, more studies are required to clarify their definite role in tumor development.
\end{abstract}

This article is freely accessible online.

Correspondence to: Dr. Sheng-Hong Tseng, Department of Surgery, National Taiwan University Hospital and National Taiwan University College of Medicine, 7 Chung-Shan S. Rd., Taipei 100, Taiwan, R.O.C. Tel: +886 223123456 (ext. 65110), Fax: +886 289665567, e-mail: shenghongtseng@gmail.com

Key Words: CPEB, tumorigenesis, microRNA, cancer, review.

\section{Translation and Cytoplasmic Polyadenylation Element Binding Proteins (CPEBs)}

Regulated mRNA translation plays an important role in normal cellular functions $(1,2)$. The translation of an mRNA is divided into three steps: initiation, elongation and termination (1). Among them, translation initiation is the rate-limiting, the most complex and the main target for translational control mechanisms (3). The 5'end of all transcribed mRNAs contains a 5 'cap and the other end is blocked by a long stretch, usually in the order of 200-500 nucleotides of adenine residues (poly(A) tail) (1). Both the cap and the poly(A) tail of mRNAs act synergistically to facilitate translation (1). The translation is affected by a number of factors that stimulate or inhibit the translation of specific mRNAs $(1,2)$. Among these factors, RNAbinding proteins that recognize specific motifs in the 3 , untranslated region (3'UTR) of their targets, are important $(1,2,4)$. Of these RNA-binding proteins, cytoplasmic polyadenylation element binding protein (CPEB) family are sequence-specific RNA-binding proteins and the key factors controling the elongation of the poly(A) tail and polyadenylation-induced translation $(2,5,6)$. CPEB binds the cytoplasmic polyadenylation element (CPE) in the 3 , untranslated regions of responding mRNAs (2, 7-9). CPEB-mediated effects on translation require at least two CPEs separated by less than 50 nucleotides in the target mRNA, which indicates the formation of CPEB-dimer (10). CPEBs can recruit the translational repression or cytoplasmic polyadenylation machineries to their target mRNAs and nucleate a complex of factors that regulates poly(A) elongation through a deadenylating enzyme (1, 4, 7, 11-13). 
The CPEB proteins are composed of 4 subtypes (CPEB1-4) where CPEB2-4 are closely related and CPEB1 is the most distant member of the family (12-14). All CPEB proteins have a similar structure with the C-terminal regions composed of two RNA recognition motifs (RRMs) and two zinc-fingercontaining sequence-specific RNA-binding proteins, as well as a regulatory $\mathrm{N}$-terminal region $(1,9,12)$. The $\mathrm{N}$-terminals of CPEB1-4 are highly variable, whereas the C-terminals are more conserved (1). CPEBs mediate many physiological and pathological processes, including germ-cell development, cell division, cellular senescence, cell differentiation, synaptic plasticity, learning and memory $(1,4,7,11-13)$. CPEBs regulate the translation of maternal mRNAs controlling meiotic cellcycle progression (15). However, CPEBs are also expressed, in various combinations, in somatic cells (15). CPEB1, 2 and 4 are essential for mitotic cell division, but CPEB3 is the only member dispensable for mitotic cell division (15). CPEB1, 2 and 4 have sequential non-redundant functions that promote the phase-specific polyadenylation and translational activation of CPE-regulated transcripts in the mitotic cell cycle, with CPEB1 being required for prophase entry, CPEB2 for metaphase and CPEB4 for cytokinesis (15).

Various CPEB subtypes may interact with each other. For example, there is evidence showing that CPEB1 and CPEB4 are functionally exchangeable, targeting the same CPE-containing mRNAs (16). Cell-cycle progression requires phase-specific regulation of the expression of multiple genes, while both CPEB1 and CPEB4 are required for cell proliferation and mitotic entry (7). The $C P E B$ knockdown using short hairpin RNA (shRNA) in HeLa cells induces proliferation defects that are modest for the CPEB4 knockdown, stronger for the CPEB1 knockdown and strong still for the double knockdown (7). In addition, there is a delay in $\mathrm{G} 1$ entry in $C P E B 1$ or $C P E B 1$ plus CPEB4 knockdown HeLa cells, which indicates a defect of in the progression through $\mathrm{G}_{2} / \mathrm{M}$ phases or the transition from $\mathrm{G}_{2} / \mathrm{M}$ to $\mathrm{G}_{1}$ (7). The interaction between CPEB1 and CPEB4 has also been noted in the regulation of angiogenesis. Increased levels of CPEB1 and CPEB4 are noted in liver tissues of cirrhotic patients and the livers and mesenteries of rats and mice with cirrhosis or/and portal hypertension, as compared with control tissues (17). In addition, CPEB4 overexpression promotes cytoplasmic polyadenylation of vascular endothelial growth factor $(V E G F)$ mRNA, increasing its translation in cirrhotic liver tissues from patients (17). The interaction between CPEB1 and CPEB4 in angiogenesis is considered through the regulation of CPEB4 by CPEB1, CPEB4 auto-amplification loop and induction of VEGF overexpression by CPEB4 (17).

\section{CPEBs and Cancer}

Cancer development is closely related to the reprogramming of gene expression and genetic derangement contributes to cell proliferation, apoptosis, metabolism, invasion and malignant transformation $(18,19)$. CPEBs control cellular proliferation, chromosome segregation and cell differentiation; aberrant expression of CPEBs correlates with certain types of cancer, indicating that cytoplasmic RNA 3' end processing is important in the control of tumor cell growth $(1,19,20)$. Several CPEBregulated mRNAs regulate cell-cycle progression, senescence and cell polarity (20). For example, the antiproliferative protein, Tob, a component of the Caf1-Ccr4 deadenylase complex, is involved in the regulation of the expression of the proto-oncogene myc (21). The $c$-myc mRNA contains CPEs in its 3'-UTR, which are recognized by the CPEB (21). CPEB recruits Caf1 deadenylase through interaction with Tob to form a ternary complex, CPEB-Tob-Caf1, and negatively regulates the expression of $c-m y c$ by accelerating the deadenylation and decay of its mRNA (21). In quiescent cells, $c$-myc mRNA is destabilized by CPEB-Tob-Caf1, while in cells stimulated by the serum, both Tob and Caf1 are released from CPEB and cmyc expression is induced early after stimulation (21). Proper balance between different isoforms of CPEBs is required to determine the cell fate between senescence and proliferation, while ectopic or unbalanced expression of the CPEB subtypes may contribute to tumor development $(1,15,19)$. There is a report mentioning that CPEB1 is down-regulated in cancers of the reproductive system, CPEB 1-3 are down-regulated in brain cancers, CPEB 3 and CPEB4 are down-regulated in cancers of the digestive system and CPEB4 is down-regulated in blood cell cancers (5); however, other reports showed contradictory results $(1,15,19,20,22,23)$. Therefore, thorough understanding the characteristics of CPEBs it is important to clarify the role of various CPEBs in tumorigenesis and develop strategies for tumor therapy.

\section{CPEB1}

CPEB 1 is known to be expressed in the brain, ovary, kidney and lung (19). It regulates germ cell development, synaptic plasticity and cellular senescence; CREB 1 is important for both meiotic progression and mitotic divisions $(5,24)$. CPEB1 neutralizing antibody or dominant-negative mutant forms of this protein inhibit cell division $(2,25)$. CPEB1 also plays a role in the regulation of the mRNAs of several encoded proteins involved in insulin signaling (24). Both phosphatase and tensin homolog (PTEN) and signal transducer and activator of transcription 3 (STAT3) are negative regulators of insulin action; and CPEB1 directly regulates the translation of PTEN and STAT3 mRNAs in human HepG2 hepatocellular carcinoma (HCC) cells (24). CPEB1-knockout mice have increased expression of PTEN and STAT3 and decreased insulin signaling to protein kinase B (Akt) in the livers of mice (24).

Senescence is a process that limits the number of times a cell divides $(12,26)$. It can be induced by many environmental stresses, such as reactive oxygen species, ultraviolet irradiation, 
DNA damage or stress $(12,26)$. Senescent cells do not divide and they express high levels of tumor suppressors p53, $\mathrm{p} 19^{\mathrm{INK} 4 \mathrm{~A}}$ and $\mathrm{p} 19^{\mathrm{ARF}}$ (27). Thus, senescence acts like a tumor suppressor to prevent malignant transformation and contribute to organizing aging (2). CPEB1 also regulates cell senescence $(1,2)$. The CPEB1-mediated down-regulation of translation is necessary for cells to become senescent (2). Absence of CPEB1 causes immortalization of mouse embryonic fibroblasts (MEFs), while the re-introduction of CPEB1 into the knockout MEFs using retroviral vector rescues senescence (12). CPEB1 requires $\mathrm{p} 53, \mathrm{p} 19^{\mathrm{ARF}}$ and $\mathrm{p} 16^{\mathrm{ARF}}$ to induce senescence, since exogenous CPEB1 could not induce senescence in $\mathrm{p} 53$, p19 $\mathrm{ARF}$ or $\mathrm{p} 16^{\mathrm{ARF}} / \mathrm{p} 19^{\mathrm{ARF}}$ double knockout MEFs (2). Reduced $p 53$ mRNA polyadenylation and translation cause senescence bypass in CPEB1-depleted cells (28). Furthermore, CPEB1 also controls the cytoplasmic polyadenylation of human $P 53$ mRNA by elongation of its poly(A) tail, while CPEBI knockdown results in deficient polyadenylation of $p 53$ mRNA and about $50 \%$ reduction of $\mathrm{p} 53$ protein levels $(1,28)$. These results suggest CPEB1 plays a significant role for inducing cellular senescence and its functions are closely related to the functions of $\mathrm{p} 53, \mathrm{p} 19^{\mathrm{ARF}}$ or $\mathrm{p} 16^{\mathrm{ARF}} / \mathrm{p} 19^{\mathrm{ARF}}$. CPEB1 also interacts with $m y c$, which is also a $C P E$-containing mRNA (2). $M y c$ is a proto-oncogene and $m y c$ mRNA has been found to coimmunoprecipitate with CPEB1 from wild-type MEFs (2). In immortal $C P E B I$ knockout MEFs, myc mRNA translation is elevated to two- to three-fold and the cells bypass senescence; in contrast, myc knockdown in immortal CPEBI knockout MEFs abrogates cell division (2). Thus, the translational repression of $m y c$ by CPEB1 is a key event in promoting senescence in MEFs (2). Similarly, in human lung and skin fibroblast primary culture, absence of CPEB1 causes a bypass of senescence accompanied by extended cellular life span, which indicates CPEB1 is required to stimulate senescence $(2,28,29)$.

CPEB1 levels are decreased in several types of human tumors, including ovary, stomach and breast cancers, myeloma, as well as colorectal and gastric cancer cell lines and myeloma cell lines $(1,20,22,30,31)$. The reduced levels of CPEB1 have also been associated with the capacity of these malignant cells to promote invasion and angiogenesis $(22,32)$. In the absence of CPEB1, mammary epithelial cells lose cell polarity (33). The loss of cell polarity can lead to epithelialmesenchymal transition (EMT) and the cells dedifferentiate and become metastatic (34). The CPEB1-depleted mammary epithelial cells alter their gene expression profile in a manner consistent with an EMT and also become motile, which are made particularly robust when cells are treated with transforming growth factor- $\beta$, an enhancer of EMT (33). Twist, a transcription factor, plays an important role in EMT and metastasis (35). CPEB1 interacts with Twistl mRNA and down-regulates its expression; in the absence of CPEB1, Twist 1 mRNA translation is elevated, which leads to EMT and metastasis $(5,36)$. In addition, upon CPEB1 reduction, matrix metalloproteinase 9 (MMP-9) mRNA, which encodes a metastasis-promoting factor, undergoes poly(A) lengthening and enhanced translation (33). In human breast cancer cells that become progressively more metastatic, CPEB1 is reduced, while MMP-9 becomes abundant (33). CPEB1-depleted mammary cells become metastatic to the lung following injection into mouse fat pads, while ectopically expressed CPEB1 prevents metastasis (33). All these results suggest the reduced CPEB1 levels might be related to the tumor formation and metastasis of the transformed breast cells (5). In addition, the CPEB1 knockout animals have significantly faster papilloma formation in carcinogenesis assay than in wild-type animals (2). For gastric cancer, CPEB1 has been noted to be down-regulated in gastric cancer cell lines and in 11 of 12 primary gastric cancer tumors (22). Down-regulation of CPEB 1 by methylation of CPEBI promoter is associated with diffuse gastric cancer, lymph node metastasis and invasion; overexpression of CPEB1 impairs these processes (22).

Glioma stem cells (GSCs) have a potency of self-renewal and multi-lineage differentiation while playing an important role in glioma initiation, growth and recurrence (37-40). Eliminating GSCs from the tumor has been considered an effective therapeutic strategy (37-40). CPEB1 can induce the differentiation of GSCs into astrocytes, with glial fibrillary acid protein (GFAP) expression being positively correlated with CPEB1 expression (32). CPEB1 overexpression reduces the expression of Nestin and Notch intracellular domain (NICD), as well as the efficiency of sphere formation (32). In contrast, CPEB 1 depletion from CSC2 glioma stem cells significantly increases Nestin expression, Notch1 cleavage and the efficiency of sphere formation (32). CPEB1 overexpression inhibits tumorigenicity of CSC2 glioma stem cells in nude mice and the CPEB1 expression negatively correlates with overall survival in glioma patients (32). In addition, overexpression of CPEB1 in rat CNS-1 glioblastoma cells inhibits the migration and tumor size on brain slices (41). These results indicate that CPEB 1 may suppress GSC self-renewal by inducing differentiation and down-regulates stemness marker expression, tumorigenesis and infiltrating phenotypes in vivo (32).

CPEB1 also regulates angiogenesis. Hypoxia is an important factor to activate tumor cell proliferation and angiogenesis; tumor cells express hypoxia-inducible factor $1 \alpha$ (HIF-1 $\alpha$ ) during hypoxia (42). Overexpression of CPEB 1 under nonstimulated conditions can repress $H I F-1 \alpha$ mRNA translation in neuroblastoma cells (42). In addition, in chick embryo chorioallantoic membrane assay, down-regulation of CPEB1 by methylation of CPEB1 promoter in KATOIII gastric cancer cells is associated with increased angiogenesis (22). Overexpression of CPEB1 inhibits angiogenesis and decreases vascular endothelial growth factor A (VEGFA) and MMP14 mRNA levels (22). 
Taken together, the data mentioned above suggest that CPEB1 is a potential tumor suppressor and lack of CPEB1 increases susceptibility to cancer.

\section{CPEB2}

CPEB2 is expressed in the brain, testis and liver $(13,19)$. It interacts with $\beta$-catenin and $\mathrm{Ca}^{2+} /$ calmodulin-dependent protein kinase II (CaMKII) (both are also CPEB1 targets); in primary hippocampal cultures, the subcellular localization of CPEB2 in neurons and astrocytes resembles that of CPEB1 (13). Therefore, there is an overlap in RNA binding specificity between CPEB1 and CPEB2 (13). Both CPEB1 and CPEB2 can affect the HIF-1 $\alpha$ protein levels and HIF activity as there is an interaction of CPEB1 and CPEB2 with the 3'-UTR of $H I F-1 \alpha$ mRNA $(42,43)$. Overexpression of CPEB1 and CPEB2 under non-stimulated conditions can repress $H I F-1 \alpha$ mRNA translation in SK-N-MC neuroblastoma cells (42). In contrast, stimulation of SK-N-MC neuroblastoma cells with insulin and, thus, activation of endogenous CPEBs increase the luciferase reporter gene fused to the 3'UTR of HIF-1 $\alpha$, as well as endogenous HIF-1 $\alpha$ protein levels; such increase can be abrogated by treating the cells with $C P E B 1$ or $C P E B 2$ siRNAs (42). Thus, CPEB2, in cooperation with CPEB1, regulate oxygen homeostasis since $H I F-1 \alpha$ is a transcriptional regulator of several genes involved in mammalian oxygen homeostasis (42). CPEB2 has been found to play a role in the tumorigenesis of triple-negative breast cancer (TNBC) (44). TNBC represents an anomalous subset of breast cancer with a greatly reduced 5year survival rate (44). The enhanced mortality and morbidity of TNBC are related to the high metastatic rate, which requires the acquisition of anoikis resistance (AnR), a process whereby anchorage-dependent cells become resistant to cell death induced by detachment (44). AnR TNBC cells have been found to have altered mRNA splicing of $C P E B 2$ with increased inclusion of exon 4 into the mature $\mathrm{mRNA}$ to produce the CPEB2B isoform (44). siRNA-induced down-regulation of the CPEB2B isoform can re-sensitize the AnR cell lines to detachment-induced cell death (44). In contrast, ectopic expression of CPEB2B in parental TNBC cell lines induces AnR and dramatically increases metastatic potential (44). These results suggest regulation of $C P E B 2$ mRNA splicing is an important mechanism in AnR and TNBC metastasis (44) and, thus, CPEB2B may have an oncogenic effect in TNBC. However, the role of CPEB2 in tumorigenesis is still limited and deserves further studies.

\section{CPEB3}

CPEB3 is expressed in the brain and heart (19). It mediates translational repression in neurons and confines the strength of glutaminergic synapses by translationally down-regulating the expression of multiple plasticity-related proteins (PRPs), including the N-methyl-d-aspartate receptor (NMDAR) and the postsynaptic density protein 95 (PSD95) (45, 46). CPEB3 deficiency imbalances NMDAR-activated CaMKII $\alpha$ signaling, which, consequently, fails to repress synaptic strength under stimulation conditions (46). In addition, $C P E B 3$ knockout mice exhibit hippocampus-dependent abnormalities that include longterm spatial memory and short-term acquisition and extinction of contextual fear memory (46). In neurons, the activation of NMDARs accumulates CPEB3 in the nucleus and redistributes CPEB3 in the nucleocytoplasmic compartments to control gene expression (47). When translocated into the nucleus, CPEB3 binds to STAT5b and down-regulates STAT5b-dependent transcription (47). CPEB3 is also related to tumorigenesis (48). It has been found to be down-regulated in colorectal cancer through the microarray-based high throughput screening (48). CPEB3 is also down-regulated in human papilloma virus (HPV)-positive cervical cancers compared with normal tissue; this down-regulation of CPEB3 is considered to occur before integration of HPV genome (30). Because CPEB3 is downregulated in colorectal cancer and HPV-positive cervical cancer, it is considered to exert tumor suppression effects. However, the data in the literature are limited and more investigations are needed to elucidate the definite role of CPEB3 in cancers.

\section{CPEB4}

CPEB4 is expressed in the brain, heart, kidney and lung (19). CPEB4-associated mRNAs are significantly enriched in multiple cellular functions that are relevant to tumorigenesis, including Ras-related molecules, cell signaling components, chromatin remodeling proteins, cyclins, apoptosis-related molecules, stress and inflammation factors, metabolic enzymes and genes related to cell migration and metastasis (19). In addition, CPEB4 has been reported in association with tumor growth, vascularization and invasion in invasive ductal breast carcinoma (IDC), colorectal cancer, pancreatic ductal adenocarcinoma, metastatic prostate cancer and glioblastoma $(19,49-52)$. High expression of CPEB4 has been observed in $48.6 \%$ of IDC samples and is possibly related to increased histological grading and $\mathrm{N}$ stage of IDC (50). IDC patients with high CPEB4 expression show shorter overall survival as compared with IDC patients with low CPEB4 expression (50). In addition, $C P E B 4$ mRNA expression is higher in the metastatic prostate cancer compared to primary cancer (51). CPEB4 is overexpressed in highly metastatic PC3 prostate cancer cell line compared with stromal and epithelial tissues from benign prostatic hyperplasia (51). Further, CPEB4 levels is negatively correlated with epithelial marker CDH1 and positively correlated with mesenchymal markers (SNAL1 and ZEB2) in prostate cancer cells (51).

Colorectal cancer tissues highly express CPEB4 and high CPEB4 expression is correlated with advanced tumor stage, lymph node metastasis, distant metastasis and poor 
prognosis in patients with colorectal cancers (52). CPEB4 is also highly expressed in the peripheral blood of colorectal cancer patients (53) and CPEB4 expression is positively associated with expression of $\mathrm{Ki}-67$, a marker for proliferative cancer cells (52). Suppression of CPEB4 expression by siRNA in SW480 and LOVO colon cancer cells enhances apoptosis and decreases cellular proliferation and is associated with decreased expression of antiapoptotic protein $\mathrm{Bcl}-\mathrm{X}$ and increased expression of proapoptotic protein Bax (52). CPEB4 is also overexpressed in glioblastomas but not expressed in normal astrocytes or paratumor tissues $(19,49)$. CPEB4 down-regulation in glioma cells results in reduced tumor size, cellular proliferation and microvessel density, as well as increased apoptosis $(19,49,54)$. In addition, glioma cells with CPEB4 suppression have reduced vimentin expression and decreased invasiveness than the control cells, thus indicating that high CPEB4 expression in astrocytic tumors correlates with a clinically aggressive feature $(49,54)$. Moreover, CPEB4 also regulates the glioma stem cells, whereas suppression of CPEB4 expression inhibits the growth, proliferation and invasion of glioma stem cells (54).

CPEB4 is overexpressed in pancreatic ductal adenocarcinoma (PDA) (19). The frequency of increased expression of CPEB 4 is $34.6 \%$ in low-grade pancreatic intraepithelial neoplasias (PanINs), $71.4 \%$ in high-grade PanINs and $88.8 \%$ in moderately or well differentiated PDAs; this frequency is relatively low in undifferentiated PDAs (36.3\%) (19). CPEB4 suppression using siRNA in RWP-1 pancreatic cancer cells reduces Matrigel invasion and soft-agar colony formation (19). In addition, CPEB4 downregulation using siRNA in pancreatic tumor cells slows tumor growth and decreases the capability to form tumors in non-pancreatic tissues, resulting in a slower progression to death in mice (19). Furthermore, the pancreatic tumor with CPEB4 knockdown has a decreased microvessel density and increased stromal formation in mice, as compared with the controls (19). Tissue plasminogen activator (tPA) has a role in tumor proliferation, migration, invasion and angiogenesis. tPA has been noted to be absent in normal pancreas and is overexpressed in $85 \%$ of PDA (19). Down-regulation of CPEB4 can induce a shorter poly(A) tail in $t P A$ mRNA and decrease tPA protein expression without affecting $t P A$ mRNA levels in PWP-1 pancreatic ductal cancer cells (19). Further, suppression of CPEB4 using shRNA in RWP-1 cells reduces the tPA expression in the subcutaneous or intraperitoneal RWP-1 xenografts in mice (19). Ectopic expression of tPA in xenografted RWP-1 cells depleted of CPEB4 increases the number of tumors in mice (19). In addition to tPA, the prooncogenic functions of CPEB4 in pancreatic cancers also affect several mRNAs associated with tumorigenesis, such as SMAD3, Bcl2 and MMP-7, suggesting CPEB4 regulates cancer progression in different ways (19).
As aberrant high expression level of CPEB4 has been demonstrated in a variety of malignancies, CPEB4 appears to exert pro-oncogenic effects to affect tumor growth, invasion and vascularization (19). However, the functions of CPEB4 in non-small cell lung cancer (NSCLC) seem to be different (55). The CPEB4 expression is relatively weak in NSCLC samples and relatively high in non-cancerous adjacent tissues (55). In addition, the CPEB4 expression is closely correlated with the overall survival of NSCLC patients, with high CPEB4 being correlated with better survival in NSCLC patients (55). Further, knockdown of $C P E B 4$ can promote the migration and invasion of NSCLC cells (55). The downregulation of CPEB4 in NSCLC disapproves the concept of pro-oncogenic effects of CPEB4 (55). Such contradiction is considered related to the various downstream targets modulated by CPEB4 in different kinds of cells (55). Certainly, more studies are needed to clarify these issues.

\section{CPEBs and microRNA}

MicroRNAs (miRNAs) are small non-coding RNAs with 19-23 nucleotides in length found in all mammalian cells. These miRNAs are incorporated into the RNA-induced silencing complex and then target the 3'-UTR (untranslated region) of a specific mRNA by a seed sequence that is located near the 5' region of the miRNA. The consequences of miRNA binding are that the mRNA is silenced or degraded, resulting in a reduced expression level of the protein encoded by the targeted mRNA (55-57). miRNAs regulate genes involved in various pathways, such as cell death, cell proliferation, stress response and metabolism (55-57). Because all $C P E B$ mRNAs contain multiple miRNA binding sites, miRNAs are also considered to regulate the functions of CPEBs $(12,58)$. Cyclin E1 is a member involved in cell-cycle transition (59). The cyclin E1 (CCNE1) mRNA has two CPEs and two miR-15/16 target sites within the 3'UTR (59). Experiments using competitor RNA and mutated cyclin E1 3'UTRs suggest cooperation of CPEB and miR-15/16 to sustain repression of the cyclin E1 mRNA during early stages of maturation in Xenopus oocyte, which indicates cyclin E1 is coregulated by CPEB and miR-15/16 (59). In addition, miR122 has been found to control $C P E B 1$ mRNA translation (60), while miR-26 and miR-92 regulate $C P E B 2$ mRNA stability (58). CPEB3 is also regulated by miRNA. miR107 is up-regulated and CPEB3 is down-regulated in HCC tissues compared with non-tumor tissues $(6,20)$. In addition, miR-107 overexpression in the Huh7 and HepG2 HCC cells can down-regulate the CPEB3 expression by directly targeting the CPEB3 3'-UTR (6). The miR-107induced down-regulation of CPEB3 is accompanied by the up-regulation of epidermal growth factor receptor (EGFR) and phospho-Akt, as well as down-regulation of p21 (6). 
Table I. The function of CPEB subtypes in various cancer types.

\begin{tabular}{|c|c|c|c|c|c|}
\hline Cancer types & CPEB1 & CPEB2 & CPEB3 & CPEB4 & References \\
\hline Gastric cancer & - & & & & 22 \\
\hline Colorectal cancer & - & & - & + & $22,48,52$ \\
\hline Pancreatic cancer & & & & + & 19 \\
\hline Breast cancer & - & + & & + & $22,33,44,50$ \\
\hline Ovarian cancer & - & & & & 30 \\
\hline Cervical cancer & & & - & & 30 \\
\hline Prostate cancer & & & & + & 51 \\
\hline NSCLC & & & & - & 55 \\
\hline Glioma & - & & & + & $19,32,49,54$ \\
\hline Myeloma & - & & & & 31 \\
\hline
\end{tabular}

CPEB, Cytoplasmic polyadenylation element binding protein; NSCLC, non-small cell lung cancer. -: tumor suppression. +: tumor promotion.

miR-107 overexpression can promote the proliferation of HepG2 and Huh7 HCC cells, enhances the migration and invasion of Huh7 cells and increases the tumor growth of subcutaneous HepG2 xenografts in nude mice (6). Restoration of CPEB3 by co-transfection of miR-107 and CPEB3 into the Huh7 cells decreases miR-107-induced proliferation and migration (6). Therefore, the miR$107 / \mathrm{CPEB} 3 / \mathrm{EGFR}$ axis is considered to play an important role in HCC progression (6).

CPEB4 has also been noted to be regulated by miRNAs in cancer. In colorectal cancer, CPEB4 exhibits prooncogenic effects and is a target of miR-203, a tumor suppressive miRNA (52). Colorectal cancers have high CPEB4 expression, that is correlated with tumor growth and patient prognosis (52). miR-203 is significantly decreased in colorectal cancers compared to adjacent normal tissues while inhibiting cancer growth and enhancing cell apoptosis (52). Transfection of miR-203 precursor in SW480 colon cancer cells inhibits CPEB4 protein expression; in contrast, transfection of miR-203 inhibitor in the SW480 cells increases the CPEB4 mRNA and protein CPEB4 expression, which indicate that CPEB4 is negatively regulated by miR203 in colorectal cancer cells (52). Further, restoration of CPEB4 can inhibit the miR-203-induced apoptosis signaling pathway, which then enhances cell proliferation and suppresses cell apoptosis (52). In NSCLC, miR-1246 is frequently up-regulated and CPEB4 is usually reduced (55). miR-1246 can promote NSCLC cell migration and invasion and $C P E B 4$ knockdown enhances cell migration and invasion, resembling the effect of miR-1246 (55). Cotransfection of miR-1246 inhibitor and siRNAs targeting CPEB4 into NSCLC A549 cells demonstrated that CPEB4 knockdown partly neutralized the suppressive effects of the miR-1246 inhibitor on NSCLC cell migration and invasion (55). These data suggest that CPEB4 is a direct and functional target of miR-1246 in NSCLC (55).

\section{Conclusion}

Regulated mRNA translation plays an important role in normal cellular functions and the CPEBs are the key factors that control the elongation of poly(A) tail during translation. These CPEBs direct coordinated programming of gene expression in the cells; however, anomalous expression of various CPEBs have been noted to be linked to the tumorigenesis, tumor growth, invasiveness and angiogenesis $(1,15,19,20,22,23)$. Different CPEBs appear to play diverse roles in cancers. The evidence from the literature suggests that CPEB1 and CPEB3 act more likely to be tumor suppressors; in contrast, CPEB2 and CPEB4 exert oncogenic effects, although there is still some controversy. In addition, a cancer type may simultaneously have the changes of the expression levels in multiple CPEB subtypes, such as colorectal cancer, breast cancer and glioma (Table I), thus indicating that different CPEB subtypes may interact with each other to affect tumorigenesis. All four $C P E B$ mRNAs contain multiple miRNA binding sites and various miRNAs can regulate different CPEB subtypes to modulate their functions (58). Therefore, manipulation of the expression of different CPEB subtypes or targeting miRNAs to regulate the CPEBs might modulate the behavior of cancer cells and be used as a treatment strategy for cancer. However, the activation of different signaling pathways by various CPEB subtypes may exert either pro-oncogenic effect or tumor suppression. The mechanisms and the signaling pathways of various CPEB subtypes in tumorigenesis are still unclear. More studies are needed to clarify their definite role in tumor development and the development of cancer therapy strategies.

\section{Acknowledgements}

This report was supported by a research grant awarded to Dr. Yun Chen from the Ministry of Science and Technology, Taiwan, R.O.C. (MOST 102-2628-B-418-001-MY3). 


\section{References}

1 Fernandez-Miranda G and Mendez R: The CPEB-family of proteins, translational control in senescence and cancer. Ageing Res Rev 11: 460-472, 2012.

2 Groisman I, Ivshina M, Marin V, Kennedy NJ, Davis RJ and Richter JD: Control of cellular senescence by CPEB. Genes Dev 20: 2701-2712, 2006

3 Jackson RJ: Alternative mechanisms of initiating translation of mammalian mRNAs. Biochem Soc Trans 33: 1231-1241, 2005.

4 MacNicol MC, Cragle CE, Arumugam K, Fosso B, Pesole G and MacNicol AM: Functional integration of mRNA translational control programs. Biomolecules 5: 1580-99, 2015.

5 Ivshina M, Lasko P and Richter JD: Cytoplasmic polyadenylation element binding proteins in development, health, and disease Annu Rev Cell Dev Biol 30: 393-415, 2014.

6 Zou CD, Zhao WM, Wang XN, Li Q, Huang H, Cheng WP, Jin JF, Zhang H, Wu MJ, Tai S, Zou CX and Gao X: MicroRNA107: a novel promoter of tumor progression that targets the CPEB3/EGFR axis in human hepatocellular carcinoma. Oncotarget 7: 266-278, 2016.

7 Novoa I, Gallego J, Ferreira PG and Mendez R: Mitotic cellcycle progression is regulated by CPEB 1 and CPEB4-dependent translational control. Nat Cell Biol 12: 447-456, 2010.

8 O'Connell ML, Cavallo WC Jr and Firnberg M: The expression of CPEB proteins is sequentially regulated during zebrafish oogenesis and embryogenesis. Mol Reprod Dev 81: 376-387, 2014

9 Schelhorn C, Gordon JM, Ruiz L, Alguacil J, Pedroso E and Macias MJ: RNA recognition and self-association of CPEB4 is mediated by its tandem RRM domains. Nucleic Acids Res 42 : 10185-10195, 2014.

10 Pique M, Lopez JM, Foissac S, Guigo R and Mendez R: A combinatorial code for CPE-mediated translational control. Cell 132: 434-448, 2008.

11 Afroz T, Skrisovska L, Belloc E, Guillen-Boixet J, Mendez R and Allain FH: A fly trap mechanism provides sequence-specific RNA recognition by CPEB proteins. Genes Dev 28: 1498-1514, 2014.

12 Richter JD: CPEB: a life in translation. Trends Biochem Sci 32: 279-285, 2007.

13 Turimella SL, Bedner P, Skubal M, Vangoor VR, Kaczmarczyk L, Karl K, Zoidl G, Gieselmann V, Seifert G, Steinhäuser C, Kandel E and Theis M: Characterization of cytoplasmic polyadenylation element binding 2 protein expression and its RNA binding activity. Hippocampus 25: 630-642, 2015.

14 Wang XP and Cooper NG: Comparative in silico analyses of cpeb1-4 with functional predictions. Bioinform Biol Insights 4: $61-83,2010$

15 Giangarra V, Igea A, Castellazzi CL, Bava FA and Mendez R: Global analysis of CPEBs reveals sequential and nonredundant functions in mitotic cell cycle. PLoS One 10: e0138794, 2015.

16 Igea A and Mendez R: Meiosis requires a translational positive loop where CPEB 1 ensues its replacement by CPEB4. EMBO J 29: 2182-2193, 2010

17 Calderone V, Gallego J, Fernandez-Miranda G, Garcia-Pras E, Maillo C, Berzigotti A, Mejias M, Bava FA, Angulo-Urarte A, Graupera M, Navarro P, Bosch J, Fernandez M and Mendez R: Sequential functions of CPEB1 and CPEB4 regulate pathologic expression of vascular endothelial growth factor and angiogenesis in chronic liver disease. Gastroenterology 150: 982-997, 2016.

18 Hanahan D and Weinberg RA: The hallmarks of cancer. Cell 100: 57-70, 2000.

19 Ortiz-Zapater E, Pineda D, Martinez-Bosch N, FernándezMiranda G, Iglesias M, Alameda F, Moreno M, Eliscovich C, Eyras E, Real FX, Méndez R and Navarro P: Key contribution of CPEB4-mediated translational control to cancer progression. Nat Med 18: 83-90, 2011.

20 D'Ambrogio A, Nagaoka K and Richter JD: Translational control of cell growth and malignancy by the CPEBs. Nat Rev Cancer 13: 283-290, 2013.

21 Ogami K, Hosoda N, Funakoshi Y and Hoshino S: Antiproliferative protein Tob directly regulates c-myc protooncogene expression through cytoplasmic polyadenylation element-binding protein CPEB. Oncogene 33: 55-64, 2014.

22 Caldeira J, Simoes-Correia J, Paredes J, Pinto MT, Sousa S, Corso G, Marrelli D, Roviello F, Pereira PS, Weil D, Oliveira C, Casares F and Seruca R: CPEB, a novel gene silenced in gastric cancer: a Drosophila approach. Gut 61: 1115-1123, 2012.

23 Rhodes DR, Kalyana-Sundaram S, Mahavisno V, Varambally R, Yu J, Briggs BB, Barrette TR, Anstet MJ, Kincead-Beal C, Kulkarni P, Varambally S, Ghosh D and Chinnaiyan AM: Oncomine 3.0: genes, pathways, and networks in a collection of 18,000 cancer gene expression profiles. Neoplasia 9: 166-180, 2007.

24 Alexandrov IM, Ivshina M, Jung DY, Friedline R, Ko HJ, Xu M, O'Sullivan-Murphy B, Bortell R, Huang YT, Urano F, Kim JK and Richter JD: Cytoplasmic polyadenylation element binding protein deficiency stimulates PTEN and Stat3 mRNA translation and induces hepatic insulin resistance. PLoS Genet 8: e1002457, 2012.

25 Mendez Z, Barnard D and Richter JD: Differential mRNA translation and meiotic progression require Cdc2-mediated CPEB destruction. EMBO J 21: 1833-1844, 2002.

26 Ben-Porath I and Weinberg RA: The signals and pathways activating cellular senescence. Int J Biochem Cell Biol 37: 961976, 2005.

$27 \mathrm{Kim}$ WY and Sharpless NE: The regulation of INK4/ARF in cancer and aging. Cell 127: 265-275, 2006.

28 Burns DM and Richter JD: CPEB regulation of human cellular senescence, energy metabolism, and p53 mRNA translation. Genes Dev 22: 3449-3460, 2008.

29 Groppo R and Richter JD: CPEB control of NF-kB nuclear localization and interleukin-6 production mediates cellular senescence. Mol Cell Biol 31: 2707-2714, 2011.

30 Hansen CN, Ketabi Z, Rosenstierne MW, Palle C, Boesen HC and Norrild B: Expression of CPEB, GAPDH and U6snRNA in cervical and ovarian tissue during cancer development. APMIS 117: 53-59, 2009.

31 Heller G, Schmidt WM, Ziegler B, Holzer S, Mullauer L, Bilban M, Zielinski CC, Drach J and Zochbauer-Muller S: Genome-wide transcriptional response to 5-aza-2'-deoxycytidine and trichostatin A in multiple myeloma cells. Cancer Res 68: 44-54, 2008.

32 Yin J, Park G, Lee JE, Park JY, Kim TH, Kim YJ, Lee SH, Yoo $\mathrm{H}$, Kim JH and Park JB: CPEB1 modulates differentiation of glioma stem cells via down-regulation of HES1 and SIRT1 expression. Oncotarget 5: 6756-69, 2014.

33 Nagaoka K, Fujii K, Zhang H, Usuda K, Watanabe G, Ivshina $\mathrm{M}$ and Richter JD: CPEB1 mediates epithelial-to-mesenchyme transition and breast cancer metastasis. Oncogene 35: 2893-901, 2015. 
34 Tam WL and Weinberg RA: The epigenetics of epithelialmesenchymal plasticity in cancer. Nat Med 19: 1438-1449, 2013.

35 Yang J, Mani SA, Donajer JL, Ramaswamy S, Itzykson RA, Come C, Savagner P, Gitelman I, Richardson A and Weinberg RA: Twist, a master regulator of morphogenesis, plays an essential role in tumor metastasis. Cell 117: 927-939, 2004.

36 Nairismagi ML, Vislovukh A, Meng Q, Kratassiouk G, Beldiman C, Petretich M, Groisman R, Fuchtbauer EM, HarelBellan A and Groisman I: Translational control of TWIST1 expression in MCF-10A cell lines recapitulating breast cancer progression. Oncogene 31: 4960-4966, 2012.

37 Galli R, Binda E, Orfanellui U, Cipelletti B, Gritti A, De Vitis $\mathrm{S}$, Fiocco R, Foroni $\mathrm{C}$, Dimeco $\mathrm{F}$ and Vescovi A: Isolation and characterization of tumorigenic, stem-like neural precursors from human glioblastoma. Cancer Res 64: 7011-7021, 2004.

38 Hemmati HD, Nakano I, Lazareff JA, Masterman-Smith M, Geschwind DH, Bronner-Fraser M and Kornblum HI: Cancerous stem cells can arise from pediatric brain tumors. Proc Natl Acad Sci USA 100: 15178-15183, 2003.

39 Singh SK, Clarke ID, Terasaki M, Bonn VE, Hawkins C, Squire $\mathrm{J}$ and Dirks PB: Identification of a cancer stem cell in human brain tumors. Cancer Res 63: 5821-5828, 2003.

40 Yuan X, Curtin J, Xiong Y, Liu G, Waschsmann-Hogiu S, Farkas DL, Black KL and Yu JS: Isolation of cancer stem cells from adult glioblastoma multiforme. Oncogene 23: 9392-9400, 2004.

41 Jones KJ, Korb E, Kundel MA, Kochanek AR, Kabraji S, McEvoy M, Shin CY and Wells DG: CPEB1 regulates $\beta$-catenin mRNA translation and cell migration in astrocytes. Glia 56 : 1401-1413, 2008

42 Hagele S, Kuhn U, Boning M and Katschinski DM: Cytoplasmic polyadenylation-element-binding protein (CPEB) 1 and 2 binding to the HIF-1 $\alpha$ mRNA 3'-UTR and modulate HIF-1 $\alpha$ protein expression. Biochem J 417: 235-246, 2009.

43 Chen PJ and Huang YS: CPEB2-eEF2 interaction impedes HIF$1 \alpha$ RNA translation. EMBO J 31: 959-971, 2012.

44 Johnson RM, Vu NT, Griffin BP, Gentry AE, Archer KJ, Chalfant $\mathrm{CE}$ and Park MA: The alternative splicing of cytoplasmic polyadenylation element binding protein 2 drives anoikis resistance and the metastasis of triple negative breast cancer. J Biol Chem 290: 25717-25727, 2015.

45 Huang YS, Kan MC, Lin CL and Richter JD: CPEB3 and CPEB4 in neurons: analysis of RNA-binding specificity and translational control of AMPA receptor GluR mRNA. EMBO J 25: 4865-4876, 2006.

46 Huang WH, Chao HW, Tsai LY, Chung MH and Huang YS: Elevated activation of CaMKII $\alpha$ in the CPEB3-knockout hippocampus impairs a specific form of NMDAR-dependent synaptic depotentiation. Front Cell Neurosci 8: 367, 2014.

47 Chao HW, Lai YT, Lu YL, Lin CL, Mai W and Huang YS: NMDAR signaling facilitates the IPO5-mediated nuclear import of CPEB3. Nucleic Acids Res 40: 8484-8498, 2012.
48 Wang X, Zbou C, Qiu G, Fan J, Tang H and Peng Z: Screening of new tumor suppressor genes in sporadic colorectal cancer patients. Hepatogastroenterology 55: 2039-2044, 2008.

49 Chen W, Hu Z, Li XZ, Li JL, Xu XK, Li HG, Liu Y, Liu BH, Jia WH and Li FC: CPEB4 interacts with Vimentin and involves in progressive features and poor prognosis of patients with astrocytic tumors. Tumour Biol 37: 5075-5087, 2016.

50 Sun HT, Wen X, Han T, Liu ZH, Li SB, Wang JG and Liu XP: Expression of CPEB4 in invasive ductal breast carcinoma and its prognostic significance. Onco Targets Ther 8: 3499-506, 2015.

$51 \mathrm{Xu} \mathrm{H}$ and Liu B: CPEB4 is a candidate biomarker for defining metastatic cancers and directing personalized therapies. Med Hypotheses 81: 875-877, 2013.

52 Zhong X, Xiao Y, Chen C, Wei X, Hu C, Ling X and Liu X: MicroRNA-203-mediated posttranscriptional deregulation of CPEB4 contributes to colorectal cancer progression. Biochem Biophys Res Commun 466: 206-213, 2015.

53 Chang YT, Huang CS, Yao CT, Su SL, Terng HJ, Chou HL, Chou YC, Chen KH, Shih YW, Lu CY, Lai CH, Jian CE, Lin $\mathrm{CH}$, Chen CT, Wu YS, Lin KS, Wetter T, Chang CW and Chu $\mathrm{CM}$ : Gene expression profile of peripheral blood in colorectal cancer. World J Gastroenterol 20: 14463-14471, 2014.

54 Liu HL, Huo JF, Liu ZJ and Chen XB: Interference on cytoplasmic polyadenylation element-binding proteins affects the invasion ability of glioma stem cells. Genet Mol Res 14: 13504-13510, 2015.

55 Huang W, Li H and Luo R: The microRNA-1246 promotes metastasis in non-small cell lung cancer by targeting cytoplasmic polyadenylation element-binding protein 4. Diagn Pathol 10: $127,2015$.

56 Chen Y, Tsai YH, Fang Y and Tseng SH: MicroRNA-21 regulates the sensitivity to cisplatin in human neuroblastoma cells. J Pediatr Surg 47: 1797-1805, 2012.

57 Chen Y, Tsai YH and Tseng SH: Inhibition of cyclin-dependent kinase 1 induced cell death in neuroblastoma cells through microRNA-34a-MYCN-survivin pathway. Surgery 153: 4-16, 2013.

58 Morgan M, Iaconcig A and Muro AF: CPEB2, CPEB3 and CPEB4 are coordinately regulated by miRNAs recognizing conserved binding sites in paralog positions of their 3'-UTRs. Nucleic Acids Res 38: 7698-7710, 2010.

59 Wilczynska A, Git A, Argasinska J, Belloc E and Standart N: CPEB and miR-15/16 co-regulate translation of cyclin E1 mRNA during Xenopus Oocyte maturation. PLoS One 11: e0146792, 2016.

60 Burns DM, D'Ambrogio A, Nottrott S and Richter JD: CPEB and two poly(A) polymerases control miR-122 stability and p53 mRNA translation. Nature 473: 105-108, 2011.

Received May 12, 2016

Revised June 7, 2016

Accepted June 8, 2016 